\title{
On closed anti de Sitter spacetimes
}

\author{
Abdelghani Zeghib \\ CNRS, UMPA, École Normale Supérieure de Lyon, 46, allée d'Italie, F-69364 Lyon cedex 07, France \\ (Zeghib@umpa.ens-lyon.fr)
}

Received: 18 May 1996 / Revised version: 3 January 1997

This article deals with the structure of the fundamental group of compact anti de Sitter spacetimes, i.e. Lorentz manifolds with constant negative curvature. Algebraically such a manifold is the quotient of the universal cover of the homogeneous space $S O(2, n) / S O(1, n)$ by a discrete group $\Gamma$ acting properly and co-compactly on it. This exists if and only if $n$ is even. Indeed, as this was observed by Kulkarni, $U(1, d)$ is contained in $S O(2,2 d)$, and acts properly transitively on $S O(2,2 d) / S O(1,2 d)$. It then suffices to take $\Gamma$ as a co-compact lattice in $U(1, d)$. The results of the present article give evidence to the question: in dimension $>3$, are all compact anti de Sitter spacetimes constructed in this way?

Mathematics Subject Classification (1991): 20H15, 53C50

\section{Introduction}

The "linear" anti de Sitter spacetime $\mathbf{H}^{1, n}$ of dimension $n+1$ is the connected component of the identity in the homogeneous space $S O(2, n) / S O(1, n)$, where $S O(p, q)$ denotes the special orthogonal group of a non degenerate quadratic form of type $(p, q)$ on $\mathbf{R}^{p+q}$ (see also Sect. 2 for another description).

It admits a unique (up to a constant) Lorentz metric (i.e. a pseudo-Riemannian metric of signature $-+\ldots+)$ invariant by the left action of $S O(2, n)$, which turns out to be of constant negative curvature. The universal anti de Sitter spacetime $\widetilde{\mathbf{H}^{1, n}}$ is the universal cover of $\mathbf{H}^{1, n}$. It is a Lorentz homogeneous space, with

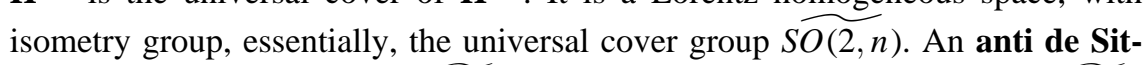
ter spacetime is a quotient $\tilde{\Gamma} \backslash \widetilde{\mathbf{H}^{1, n}}$, where $\tilde{\Gamma}$ is a discrete subgroup of $\operatorname{Isom}\left(\widetilde{\mathbf{H}^{1, n}}\right)$ acting properly and freely. We will always denote by $\Gamma$ the projection of $\tilde{\Gamma}$ in $S O(2, n)$. Such a manifold inherits a Lorentz metric of constant negative curvature. Conversely, a complete Lorentz manifolds of constant negative curvature is an anti de Sitter spacetime. 
Here we are concerned with compact anti de Sitter spacetimes. Recently, in [11], B. Klingler adapted the Carrière's completeness proof of compact flat Lorentz manifolds, to the general constant curvature case. In fact, in a previous version of the present article, we conjectured that Carrière's completeness theorem (and method) [3], may be generalized to compact locally symmetric Lorentz manifolds (see also [15] about this question). Therefore, closed anti de Sitter spacetimes are just closed manifolds of constant negative curvature. In the present article, we focus attention on the algebraic structure of the fundamental groups of such spacetimes.

As observed, for example in [14], thanks to a Gauss-Bonnet formula, even dimensional manifolds of constant non zero curvature have non zero Euler class. But this can not be the case of a compact Lorentz manifold (without any condition on its curvature), as its lightlike cone determines a continuous field of directions. Therefore compact anti de Sitter spacetimes are odd dimensional.

For odd dimensions, R. Kulkarni observed that the group $G=U(1, d)$ is contained in $S O(2,2 d)$, and thus acts isometricaly on $\mathbf{H}^{1,2 d}$. This action is in fact transitive and proper, since its isotropy group is just $U(d)$. It then follows that any co-compact lattice $\Gamma$ in $U(1, d)$ acts properly, discontinuously and cocompactly on $\mathbf{H}^{1,2 d}$. This action is free if $\Gamma$ is torsion free. The quotient $\Gamma \backslash$ $\mathbf{H}^{1,2 d}$ is thus a compact anti de Sitter spacetime. Any spacetime, obtained, up to finite covers, by this construction, will be called standard, and special standard if $\Gamma$ is contained in $S U(1,2 d)$. (See Theorem $\mathbf{B}$ and 8.1 for another causal characterizations of these spaces).

These anti de Sitter spacetimes admit the following Riemannian description. Observe that the (Riemannian) complex hyperbolic space of complex dimension $d, \mathbf{H}_{\mathbf{C}}^{d}=U(1, d) / S^{1} \times U(d)$ is obtained as a quotient of $\mathbf{H}^{1,2 d}$, by the center $S^{1}$ of $U(1, d)$ (the group of unitary complex multiplication), and that $\operatorname{Isom}^{0}\left(\mathbf{H}_{\mathbf{C}}^{d}\right)=$ $S U(1, d)$. Moreover $\mathbf{H}^{1,2 d}$ may be seen as the circle bundle associated to the canonical (or may be the anti-canonical) bundle of $\mathbf{H}_{\mathbf{C}}^{d}$. Therefore, a special standard anti de Sitter spacetime $\Gamma \backslash \mathbf{H}^{1,2 d}$ is the canonical $S^{1}$ bundle over the complex hyperbolic manifold $\Gamma \backslash \mathbf{H}_{\mathbf{C}}^{d}$. Conversely the canonical $S^{1}$ bundle of a hyperbolic complex manifold admits a structure of a special standard anti de Sitter spacetime. For hyperbolic surfaces, this construction yields the Killing Lorentz structure on their unit tangent bundle.

Here follows our principal result, it suggests that in dimension $>3$, all anti de Sitter spacetimes are standard!

Theorem A Let $M^{1, n}=\tilde{\Gamma} \backslash \widetilde{\mathbf{H}^{1, n}}$ be a compact anti de Sitter spacetime with $n>2$. Then up to finite covers, $M$ is in fact a quotient $\Gamma \backslash \mathbf{H}^{1, n}$, that is in other words, the projection $\Gamma$ of $\pi_{1}(M)$ in $S O(2, n)$, acts discontinously and properly on $\mathbf{H}^{1, n}$. Moreover, exactly one of the following two possibilities holds for $\Gamma$ :

i) $\Gamma$ is Zariski dense in $\mathrm{SO}(2, n)$.

ii) $\Gamma$ is conjugate to a (discrete) uniform lattice of $U(1, d)(n=2 d)$, i.e. $M$ is standard. 
Some comments are in order:

The 3 dimensional case. The linear anti de sitter spacetime $\mathbf{H}^{1,2}$ is identified with the group $\operatorname{SL}(2, \mathbf{R})$ endowed with its Killing form. Its isometry group is essentially the direct product $S L(2, \mathbf{R}) \times S L(2, \mathbf{R})$ acting by the left and the right.

Special standard (resp. standard) manifolds are just quotients $\Gamma \backslash S L(2, \mathbf{R})$, when $\Gamma \subset S L(2, \mathbf{R}) \times\{1\}$ (resp. $\Gamma$ is a subgroup of $S L(2, \mathbf{R}) \times S^{1}$, with the first factor acting by left multiplication and the second by the right).

It is clear that special standard anti de Sitter spacetimes are Seifert fiber spaces. A basic work on closed anti de Sitter spacetimes of dimension 3 is [13]. The authors proved that, as in the special standard case, all the closed anti de Sitter spacetimes are (topologically) seifert fiber spaces. They then asked if all of them are (geometrically) standard? This was immediately seen to be not true by B. Goldman [10], as one can non trivially deform standard spacetimes. This is essentially du to the non simplicity of $\operatorname{SO}(2,2)$, which is not true for higher dimensions. The systematic question: what kind of $\Gamma$ in $S O(2,2)$ can occur, is studied by F. Salein [19].

Finiteness of levels. The fact that, up to finite covers, one may obtain $M$ as a quotient of the "linear model" $\mathbf{H}^{1, n}$ (which means that we don't need its universal cover $\widetilde{\mathbf{H}^{1, n}}$ ), is expressed in [13] as a finiteness level property. Indeed for each integer $k, \mathbf{H}^{1, n}$ has a $k$-cover $\mathbf{H}_{k}^{1, n}$, and (up to orientability conditions) $M$ is a quotient of some $\mathbf{H}_{k}^{1, n}$. The level of $M$ is the smallest $k$.

The finiteness of levels (for closed anti de Sitter spacetimes) is a fundamental property, and is by no means obvious (it may also have a physical meaning, as a quantum number...!). The fact that the compact anti de Sitter spacetimes of dimension 3 have finite levels, is announced in [13] (and then used to prove other claims). Nevertheless, their proof is far from being convincing. Our method of proof here can easily be extended to the dimension 3 .

The Zariski closure. The Theorem states a dichotomy for the holonomy group $\Gamma$ : it is either Zariski dense, or a lattice in $U(1, d)$. However, we do not know non standard examples, that is those for which $\Gamma$ is Zariski dense.

In dimension 3, Zariski dense examples exist, and the dichotomy itself is not true. One can check this by taking small deformations of a compact lattice $\Gamma \subset S L(2, \mathbf{R})$ in $S O(2,2)=S L(2, \mathbf{R}) \times S L(2, \mathbf{R})$.

For $d>1, S O(2,2 d)$ is simple, and it is not clear how to deform (non trivially) inside it, a lattice $\Gamma \subset U(1, d)$.

In general, lattices in simple groups are locally rigid, but those of our interesting group $S U(1, d)$ (together with those of $S O(1, n)$ ) appear as exceptional cases. More precisely, by classical rigidity theorems [18], a lattice in a simple group $G$, except for $G=S O(1, n)$ or $G=S U(1, d)$ is locally rigid for any non trivial representation of $G$ (in any $S L(N, \mathbf{R})$ ). 
On the other hand, as exceptional case for rigidity, there are in fact lattices of $S U(1, d)$ which may be deformed (outside $U(1, d)$ ) in some $S L(N, \mathbf{R})$ [16]. Nevertheless, it is not obvious how to do this inside $S O(2,2 d)$ for $2 d>2$. We therefore dare ask:

Question 1.1 Are all anti de Sitter spacetimes of dimension $>3$, standard?

\subsection{Related results}

The question we are asking above, may be posed in the general context of compact quotients of homogeneous spaces, and may be expressed as a Bieberbach rigidity for these spaces (because it is somewhat reminiscent to the Bieberbach Theorem for cristalographic groups).

Consider a homogeneous space $I / H$, quotient of a connected Lie group $I$ by a connected Lie subgroup $H$. We are researching for discrete subgroups $\Gamma \subset I$ acting properly co-compactly and freely on $I / H$ (so that the quotient $\Gamma \backslash I / H$ is a compact manifold, locally modeled on $I / H)$.

As in the definition of standard anti de Sitter spacetimes, one may start by consider a radically simpler problem which is, first, find a connected Lie subgroup $G \subset I$ acting co-compactly (or say, transitively) and properly on $I / H$, and next take $\Gamma$ to be a co-compact lattice in $G$. One says that $I / H$ satisfies the Bieberbach rigidity, if all its compact quotients are of this type. As example, after many works during the last decade, the structure of compact flat Lorentz manifolds, was elucidated, as in the following Theorem, by proving a completeness result and a Bieberbach rigidity.

Theorem 1.2 ([3], [7], [8], [9],...) Let $M^{1, n}$ be a compact lorentz flat manifold. Then there is a solvable group $G$ acting isometrically and simply transitively on the Minkowski space $\mathbf{R}^{1, n}$ and a lattice $\Gamma$ in $G$ such that up to finite covers, $M=\Gamma \backslash \mathbf{R}^{1, n}(=\Gamma \backslash G)$.

There are many examples of such solvable Lie groups [9]. In contrary, in our question (above), we are hoping for a unique Bieberbach rigidity, because we suppose that only the Lie group $G=U(1, d)$ is possible. It seems that this uniqueness phenomenon is a consequence of the presence of reductive (or say semi-simple) groups.

Notice finally that the compact de Sitter spacetimes, i.e. Lorentz manifolds of constant positive curvature, are easy to understand: they do not exist at all! (see [2] and [12]). Modulo the completeness (which is now proved by [11]), the non existence of compact anti de sitter spacetimes is known as the Calabi-Markus phenomenon [2] (see also [12]).

\subsection{Further results}

In dimension 3, the beauty of anti de Sitter spacetimes is amplified by the isometric dynamical systems that they support. 
For $M=\Gamma \backslash \mathbf{H}^{1, n}$, its isometry group $\operatorname{Isom}(M)$ is identified to $\operatorname{Nor}(\Gamma) / \Gamma$, where $\operatorname{Nor}(\Gamma)$ is the normalizer of $\Gamma$ in $\operatorname{SO}(2, n)=\operatorname{ISom}\left(\mathbf{H}^{1, n}\right)$. The neutral component $I_{\operatorname{som}}(M)$ corresponds to Centralizer $(\Gamma) / \Gamma$. This component is easy to understand from the Theorem above. Nevertheless, the normalizer is typically a non algebraic group and is "difficult" to detect algebraically in the case of Zariski dense subgroups of $S O(2, n)$. Our result is:

Theorem B The isometry group of a compact anti de Sitter spacetime $M^{1, n}, n>2$, is compact. More precisely, Isom $(M)$ is finite, unless $M$ is standard, in which case, the identity component Isom ${ }^{0}(M)$ is isomorphic to $S^{1}$.

It will be explained in Sect. 10, how to deduce the Theorem above from the following one.

Theorem $\mathbf{C}$ A compact anti de Sitter spacetime of dimension $>3$, has no $C^{0}$ lightlike geodesic foliation of codimension one.

\section{Geometric and algebraic preliminaries}

The linear model of $\mathbf{H}^{1, n}$. We denote by $\mathbf{R}^{2, n}$ the space $\mathbf{R}^{2+n}$ equipped with a (non degenerate) quadratic form $q$ of signature $--+\ldots+$. Any such a form is equivalent to the standard one: $q_{0}=-x_{0}^{2}-x_{1}^{2}+x_{2}^{2}+\ldots+x_{n+1}^{2}$. A subspace $P \subset \mathbf{R}^{2+n}$ is called degenerate or lightlike if the restriction of $q$ on $P$ is degenerate (i.e. $P \cap P^{\perp} \neq 0$ ). It is called isotropic if $q$ vanishes on $P$ (i.e. $P \subset P^{\perp}$ ). Such an isotropic space has at most dimension 2.

Let $S O(2, n)$ be the special orthogonal group of $q_{0}$. From the equivalence of quadratic forms of the same signature as $q_{0}$, we deduce that $S O(2, n)$ acts transitively on each connected component of each level $q_{0}^{-1}(c)$, in $\mathbf{R}^{2+n}-\{0\}$, for $c \in \mathbf{R}$.

The anti de Sitter space $\mathbf{H}^{1, n}$ is identified with $q_{0}^{-1}(-1)$, since the last space is a homogeneous space of the group $S O(2, n)$ with isotropy group exactly $S O(1, n)$, at $(1,0, \ldots, 0)$. The Lorentz metric on $\mathbf{H}^{1, n}$ is induced from the pseudo-riemannian metric on $\mathbf{R}^{2, n}$ (defined by $q_{0}$ ).

From our (mathematical) point of view, neither the value -1 , nor the normal form $q_{0}$ have particular importance. Any sheet of a level $q^{-1}(c)$ for $c<0$ and $q$ having the same signature as $q_{0}$ may serve as a model of the anti de Sitter space.

Notice that $S O(2, n)$ acts transitively on the space of isotropic directions, as well as on the space of isotropic 2-spaces.

Lightlike geodesic hypersurfaces and foliations. A geodesic submanifold $H$ of $\mathbf{H}^{1, n}$ is obtained as a connected component of an intersection $E \cap \mathbf{H}^{1, n}$, where $E$ is a vector subspace of $\mathbf{R}^{n+2}$, with $\operatorname{dim} E=\operatorname{dim} H+1$ (see [20]). In particular, a geodesic hypersurface is obtained as $H_{u}=u^{\perp} \cap \mathbf{H}^{1, n}$ for some vector $u$. One 
verifies that $H_{u}$ is lightlike, i.e. the restriction of the metric to it is degenerate, exactly when $u$ is isotropic. Observe that in fact $H_{u}$ has two connected component, and thus any connected lightlike geodesic hypersurface equals some connected component of some $H_{u}$. However, to simplify notation, we shall argue below as if $H_{u}$ was connected, one in fact easily sees this does not matter in what follows.

Fact 2.1 $A\left(C^{0}\right)$ lightlike geodesic foliation of $\mathbf{H}^{1, n}$ is obtained as follows. There is an isotropic 2-vector space $P$ such that the leaves are the traces in $\mathbf{H}^{1, n}$ of the hyperplanes $u^{\perp}$, for $u \in P$. A lightlike geodesic foliation of $\widetilde{\mathbf{H}^{1, n}}$ is a lift of such a foliation in $\mathbf{H}^{1, n}$.

Proof. Consider two lightlike geodesic hypersurfaces of $\mathbf{H}^{1, n}, H_{u}$ and $H_{v}(u$ and $v$ are defined up to multiplicative constants). Let $P$ be the 2- vector space generated by $u$ and $v$. Since both $u$ and $v$ are isotropic, $P$ must be isotropic or Lorentzian (i.e. with restricted metric of signature -+ ). In this last case, the metric on $P^{\perp}=u^{\perp} \cap v^{\perp}$ has a signature $-+\ldots+$. Thus $P^{\perp}$ meets $\mathbf{H}^{1, n}$. Hence $H_{u} \cap H_{v}=u^{\perp} \cap v^{\perp} \cap \mathbf{H}^{1, n}$ is not empty. Hence the two geodesic subspaces $H_{u}$ and $H_{v}$ have a trivial intersection (inside $\mathbf{H}^{1, n}$ ) exactly if $u$ and $v$ are orthogonal (and of course non collinear).

Therefore a foliation is defined by a one parameter family of isotropic and mutually orthogonal vectors of $\mathbf{R}^{2, n}$. Thus they generate an isotropic vector subspace. Since the signature is $--+\ldots+$, these vectors must lie in a 2 -vector space.

We now observe that our analysis for intersection of geodesic subspaces, also holds, locally in $\widetilde{\mathbf{H}^{1, n}}$. That is for any point $x$, there is a neighborhood $V$, such that, two connected components passing through $V$ of lifts of two geodesic hypersurfaces $H_{u}$ and $H_{v}$, must meet, unless $u$ and $v$ are orthogonal.

Lie subgroups of $S O(2, n)$. Here follows some preparing elementary facts about actions on $\mathbf{H}^{1, n}$ of subgroups of $S O(2, n)$. We shall use the word rotation for a linear map $A$ on a linear space $E$, to mean that it is conjugate to a multiplication by a unit complex number on a complex space. This is equivalent to that $A$ is semi-simple, with a unique (non real) eigenvalue, which furthermore has module 1. If furthermore $A \in S O(2, n)$, then the conjugacy is inside $S O(2, n)$. One easily proves:

Fact 2.2 The centralizer of a one parameter group of rotation of $S O(2, n)$ is conjugate to $U(1, d)$, with $n=2 d$.

We say that a group (even non discrete) acts co-compactly on some space, if the iterates by this group of some compact subset, cover the whole space (in particular every continuous invariant function on that space is bounded).

Lemma 2.3 For $n>0$, the subgroup $G$ of elements of $\operatorname{SO}(2, n)$ preserving a non trivial splitting $\mathbf{R}^{n+2}=E_{1} \bigoplus E_{2}$ does not act co-compactly on $\mathbf{H}^{1, n}$. 
Proof. Assume the contrary. The form $q_{1}(x, x)=q_{0}\left(\pi_{1}(x), \pi_{1}(x)\right)$, where $\pi_{1}$ is the projection onto $E_{1}$, is another $G$-invariant form. So by co-compactness, $q_{1}$ is bounded on $\mathbf{H}^{1, n}: a \leq q_{1}(x, x) \leq b$, for $x \in \mathbf{H}^{1, n}$. Hence: $a \lambda^{2} \leq q_{1}(y, y) \leq b \lambda^{2}$, for $y \in q_{0}^{-1}\left(-\lambda^{2}\right)$.

In particular the lightlike cone $q_{0}^{-1}(0)$ is contained in that of $q_{1}$. This would imply, if $q_{1}$ is not identically 0 , that the two forms have the same lightlike cones (as both of them are connected analytic codimension 1 subsets). This is known to imply the two forms are proportional. This is impossible since $q_{1}$ is degenerate (its kernel contains $E_{2}$ ). It then follows that $q_{1}$ is trivial. The same is true for $q_{2}$, the analogous form constructed from $E_{2}$. This is impossible.

Fact 2.4 Let $G$ be a connected Lie subgroup of $S O(2, n)$ acting co-compactly on $\mathbf{H}^{1, n}$. Then, one of the following possibilities holds:

i) $G$ is semi-simple.

ii) $n=2 d$, and $G$ is conjugate to $U(1, d)\left(=S U(1, d) \times S^{1}\right)$.

iii) $G$ preserves an isotropic line.

iv) $G$ preserves an isotropic 2-vector space.

Proof. If $G$ is not semi-simple, then it has a non trivial radical $R$ (its maximal connected normal solvable subgroup). There is a root (i.e. a non trivial homomorphism) $\alpha: R \rightarrow \mathbf{C}$ and a maximal subspace $E \subset \mathbf{R}^{n+2}$, on which any element $A \in R$ acts as a multiplication by $\alpha(A)$. More precisely, if $\alpha$ is real, then $A$ is actually a multiplication by $\alpha(A)$. If $\alpha$ is complex, then the same is true for some complex structure on $E$. Furthermore $E$ is preserved by the normalizer of $G$ (in $G L(n+2))$. In particular $E$ is preserved by $G$.

A vector belonging to a characteristic space of an orthogonal matrix, associated to a non unitary eigenvalue, must be isotropic. Hence in the case $E=\mathbf{R}^{2+n}$, we have $\alpha(A) \in S^{1} \subset \mathbf{C}$, for any $A \in R$. Therefore, $R$ is embedded in $S^{1}$ and so the action of $G$ by conjugacy is trivial. That is $G$ centralizes $R$. From above, $G$ is conjugate to a subgroup of $U(1,2 d)$, with $2 d=n$.

If now $E \neq \mathbf{R}^{2+n}$, and $E$ is not degenerate, then $G$ preserves a non trivial splitting $\mathbf{R}^{2+n}=E \bigoplus E^{\perp}$, which is impossible by the Lemma 2.3. If $E$ is degenerate, then its kernel $E \cap E^{\perp}$ is $G$-invariant isotropic subspace which has therefore dimension 1 or 2 .

By considering characteristic subspaces, as in the proof above, one checks:

Fact 2.5 Let $A^{t}$ be a one parameter group of $\operatorname{SO}(2, n)$. Suppose that, $G$, the centralizer of $A^{t}$, acts co-compactly on $\mathbf{H}^{1, n}$. Then, one of the following two possibilities must hold:

i) $A^{t}$ is a one parameter rotation group, and $G$ is conjugate to $U(1, d)$, for $n=2 d$. ii) $A^{t}$ is unipotent, and $G$ preserves an isotropic subspace of dimension 1 or 2.

Parabolic subgroups. We say that a subgroup of $S O(2, n)$ is of type $\mathbf{P}_{1}$ (resp. of type $\mathbf{P}_{2}$ ) if it is the stabilizer of an isotropic line (resp. a 2-vector space). Note the following geometric and algebraic interpretations. 
Fact 2.6 i) A subgroup of $S O(2, n)$ is of type $\mathbf{P}_{1}$ (resp. $\left.\mathbf{P}_{2}\right)$ if and only if it is the stabilizer in $\mathrm{SO}(2, n)$ of a lightlike geodesic hypersurface (resp. a lightlike geodesic foliation).

ii) A maximal parabolic subgroup of $S O(2, n)$ is a group of type $\mathbf{P}_{1}$ or $\mathbf{P}_{2}$.

Proof. The point (i) follows from 2.1. For the point (ii), observe firstly, that a group of type $\mathbf{P}_{1}$ or type $\mathbf{P}_{2}$ is parabolic, because it may be seen as an isotropy group of a projective action of $S O(2, n)$ on a projective space. Conversely, a maximal parabolic subgroup of $S O(2, n)$ is not semi-simple, and is co-compact in $S O(2, n)$. In particular it acts co-compactly on $\mathbf{H}^{1, n}$. It follows from 2.4 , that it is of type $\mathbf{P}_{1}$ or $\mathbf{P}_{2}$.

\section{Plan of the proof of Theorems. Tools}

Notations and steps. Let's firstly precise that we will always suppose $n>2$. Also we will follow the notational convention: the "operator overtilde" (on notations) ensures the passage from objects in $\widetilde{\mathbf{H}^{1, n}}$ to that in $\mathbf{H}^{1, n}$. For instance if $\tilde{A}$ (resp. A) is a subgroup of $\operatorname{Isom}\left(\widetilde{\mathbf{H}^{1, n}}\right)(\operatorname{resp} . \operatorname{SO}(2, n))$, then $A(\operatorname{resp} . \tilde{A})$ is its projection in (resp. lift to) $S O(2, n)$ (resp. Isom $\left(\widetilde{\mathbf{H}^{1, n}}\right)$ ). However, if $A$ is connected, $\tilde{A}$ will be just the identity component of its inverse image in $\operatorname{Isom}\left(\widetilde{\mathbf{H}^{1, n}}\right)$. For instance if $A$ is simply connected, then we identify $\tilde{A}$ with $A$.

Let $M=\tilde{\Gamma} \backslash \widetilde{\mathbf{H}^{1, n}}$ be a compact anti de sitter spacetime, and let $G$ be the algebraic closure of $\Gamma$ (the projection of $\tilde{\Gamma}$ in $S O(2, n)$ ). It has finitely many connected components, and after passing to a finite index subgroup of $\tilde{\Gamma}$, we may suppose that $G$ is in fact connected. The compactness of $M$ implies that $G$ acts co-compactly on $\mathbf{H}^{1, n}$. Here are the steps of proofs:

1) We will start in Sect.4, by proving that $G$ is not contained in a group of type $\mathbf{P}_{1}$.

2) The key technical contribution in the present article is the impossibility of the type $\mathbf{P}_{2}$, too, that is $G$ is not contained in a group of type $\mathbf{P}_{2}$. This is the content of the Main Proposition 6.1, which proof occupies Sect. 6.

3) In view of the geometric interpretation of the type $\mathbf{P}_{2}$ (2.6), this would imply Theorem $\mathbf{C}$, that is a compact anti de Sitter spacetime has no codimension 1 lightlike geodesic foliation.

4) From $2.4, G$ may be $S O(2, n)$, conjugate to $U(1, d)$ or a semi-simple subgroup acting co-compactly on $\mathbf{H}^{1, n}$. It is the aim of Sect. 7 to show that such a subgroup is conjugate to $S U(1, d)$. This would prove half of Theorem $\mathbf{A}$, that about Zariski closures.

5) The compactness of the isometry group follows from Theorem $\mathbf{C}$, because a compact Lorentz manifold admitting a non compact isometry group, possesses a lightlike geodesic foliation, see [5], and [22]. Using this, we prove in the last section of the article the first part of Theorem A (i.e. finiteness of levels), and the last part of Theorem $\mathbf{B}$. 
Tools. The content of the steps 1 and 2 , is that the homogeneous space $\widetilde{\mathbf{H}^{1, n}}$, endowed with a restricted isometry group, corresponding to a group of type $\mathbf{P}_{1}$ or type $\mathbf{P}_{2}$, has no compact quotient. There is no general method of proving that some homogeneous space does not have a compact quotient. Our tools here consist of the two following unimodularity and nilpotency facts.

Unimodularity. The following proposition summarizes classical facts about invariant measures on locally homogeneous spaces.

Proposition 3.1 Let $G$ be a Lie group and $H$ a closed connected subgroup, with Lie algebras respectively $\mathscr{G}$ and $\mathscr{H}$. Define, for $g \in G$, the modular distortion: $\Delta_{G}(g)=\operatorname{det}(\operatorname{Ad}(g) / \mathscr{G})$, and for $g$ normalizing $H: \Delta_{H}(g)=\operatorname{det}(\operatorname{Ad}(g) / \mathscr{H})$. Then:

i) If $G / H$ admits a non trivial $G$ (left) invariant measure, then: for $h \in H$, $\Delta_{G}(h)=\Delta_{H}(h)$.

ii) If furthermore $G / H$ admits a left quotient $\Gamma \backslash G / H$, of finite measure (with $\Gamma$ discrete), then, for any $g$ normalizing $H: \Delta_{G}(g)=\Delta_{H}(g)$.

Proof. A detailed proof may be deduced following the developments of ([18], Chapter 2). Let $\bar{\omega}$ be a $p$-form on $\mathscr{G}$, with $p=\operatorname{dim} \mathscr{H}$, which is a volume form on $\mathscr{H}$. It defines a $p$-form $\omega$ on $G$, invariant by the left action.

Let $\mu$ be the given measure on $G / H$. Define on $G$, the measure $\nu$ by: $\int f d \nu=$ $\int\left(\int_{x H} f \omega\right) d \mu$, where $f$ is a continuous function with compact support in $G$. This is a Haar measure on $G$ since it is $G$-invariant by the left. In particular the measure $\mu$ is defined by means of a volume form $\alpha$ on $G / H$. Its pull-back in $G$, is a form $\beta$ which is $G$-invariant by the left, and also $H$-invariant by the right (because it comes from $G / H$ ). That is, the action of $A d(H)$ on $\mathscr{G} / \mathscr{H}$ preserves a volume form. This exactly means equality of modular distortions.

For the point (ii), notice that if $g$ normalizes $H$, then it acts on $\Gamma \backslash G / H$. Moreover, $g^{*} \mu=c \mu$ for some positive constant $c$. But if $\mu$ is finite, then necessarily $c=1$, that is $g$ preserves $\mu$. As above, $g$ must have the same distortion along $\mathscr{H}$ and $\mathscr{G}$.

Nilpotency. In the proof of the Main Proposition 6.1, we will meet groups which are neither discrete, nor closed. For this purpose, let us formulate the following notion.

Definition 3.2 Let $B$ be a subgroup of a Lie group $C$. The non-discrete part of $B$ is $B_{n d}=B \cap \bar{B}^{0}$, where $\bar{B}^{0}$ is the identity component of the closure of $B$. It is a normal subgroup of $B$, characterized as being the minimal subgroup $B^{\prime}$ of $B$ such that, $B$ projects onto a discrete subset of the coset space $C \overline{B^{\prime}}$.

From the definition, we get:

Fact 3.3 Suppose that $B_{n d}$ is contained and is co-compact in a closed normal subgroup $E$ of $C$. Then the projection of $B$ on $C / E$ is a discrete subgroup. 
The following Lemma will be a fundamental ingredient in proofs. It is a straightforward consequence of the Margulis Lemma, and seems to be presented with (essentially) the following general form, at the first time in [4].

Lemma 3.4 Let $G$ be a connected Lie group which is a semi-direct product $G=$ $N \rtimes A$, where $N$ is a nilpotent normal subgroup. Suppose that the adjoint action of A commutes with a non trivial homothety of $N$, that is a strictly contracting or a strictly expanding automorphism of $N$. (This is for instance the case if $A$ contains a central element which induces a contraction on $N$ ).

Let $\pi: G \rightarrow A$ be the projection and $\Phi \subset G$ a discrete subgroup. Set $\phi^{\prime}=\pi(\phi)$ and $\phi^{\prime \prime}=\phi_{\text {nd }}^{\prime}$ its non discrete part. Then $\phi^{\prime \prime}$ is nilpotent.

In fact, also the relative non discrete part of $\phi$, defined by $\Phi_{n d, A}=\phi \cap \pi^{-1}\left(\phi^{\prime \prime}\right)$, is nilpotent (relative here means that it is associated to the factor $A$ ).

\section{Algebraic structure of groups of type $P_{1}$}

Here we show that the group $\Gamma$ can not preserve an isotropic line, i.e. $\Gamma$ is not contained in a subgroup of type $\mathbf{P}_{1}$. Consider the quadratic form: $q=x_{0} x_{n+1}-$ $x_{1}^{2}+x_{2}^{2}+\cdots+x_{n}^{2}$. Let $P_{1}$ be the group of transformations preserving $q$ and the line $\mathbf{R} e_{0}$, where $e_{0}=(1,0 \ldots, 0)$. One verifies that $P_{1}$ is a semi-direct product of the following subgroups:

1) A semi-simple group $S_{1}$ isomorphic to $S O(1, n-1)$, consisting of matrices:

$$
\left(\begin{array}{ccc}
1 & 0 & 0 \\
0 & A & 0 \\
0 & 0 & 1
\end{array}\right), A \in S O(1, n-1) .
$$

2) A diagonal group $D_{1}$ isomorphic to $\mathbf{R}^{*}$ :

$$
\left(\begin{array}{ccc}
\lambda & 0 & 0 \\
0 & I & 0 \\
0 & 0 & \lambda^{-1}
\end{array}\right), \lambda \in \mathbf{R}^{*} .
$$

3) An unipotent subgroup $N_{1}$ isomorphic to $\mathbf{R}^{n}$ :

$$
\left(\begin{array}{ccccc}
1 & 2 a_{1} & \cdots & 2 a_{n} & -\left(-a_{1}^{2}+a_{2}^{2}+\cdots+a_{n}^{2}\right) \\
0 & 1 & \cdots & 0 & -a_{1} \\
& & \cdots & \cdot & \cdot \\
0 & & \cdots & 1 & -a_{n} \\
0 & & \cdots & 0 & 1
\end{array}\right),\left(a_{1}, \ldots, a_{n}\right) \in \mathbf{R}^{n} .
$$

Moreover, one verifies that: $S_{1}$ and $D_{1}$ commute, $N_{1}$ is normal, the adjoint action of $S_{1}$ on $N_{1} \sim \mathbf{R}^{n}$, is the usual one, and finally that $D_{1}$ acts by multiplication on $N_{1}\left(\sim \mathbf{R}^{n}\right)$. 
Action of $P_{1}$ on $\mathbf{H}^{1, n}$. Observe that $P_{1}$ has exactly two orbits in $\mathbf{H}^{1, n}$ : an open dense one: $\mathscr{O}=P_{1} e$, for $e=(1,0, \ldots, 0,-1)$, and its complementary: $\mathscr{O}^{\prime}=$ $\left(\mathbf{R} e_{0}\right)^{\perp} \cap \mathbf{H}^{1, n}$. Note that the isotropy group of $e$ is $S_{1}$ which is in fact centralized by $D_{1}$. Hence, the modular distortion of $\operatorname{Ad}\left(D_{1}\right)$ on $S_{1}$ is trivial, but obviously not that on $P_{1}=N_{1} \rtimes\left(S_{1} \cdot D_{1}\right)$. We deduce from 3.1 that the universal cover of (6) has no finite volume quotient.

The geometry of a lightlike geodesic hypersurface. Any lightlike geodesic hypersurface is congruent to $H=e_{0}^{\perp} \cap \mathbf{H}^{1, n}$, and its stabilizer in $S O(2, n)$ is conjugate to $P_{1}$.

The direction $e_{0}$ determines a 1-dimensional foliation $\mathscr{D}$ of $H, \mathscr{D}_{x}=\{x+$ $\left.r e_{0}, r \in \mathbf{R}\right\}$. Observe that the set $\left\{\left(0, x_{1}, \ldots, x_{n}, 0\right) /-x_{1}^{2}+x_{2}^{2}+\cdots+x_{n}^{2}=\right.$ $-1\}$, which may be identified to $\mathbf{H}^{n-1}$, is a global cross section of $\mathscr{D}$. In fact $\mathscr{D}$ is a transversally Riemannian foliation in the sense that the group $P_{1}$ acts isometrically on $\mathbf{H}^{n-1}$. Indeed $P_{1}$ acts via its factor $S_{1}$ which acts on $\mathbf{H}^{n-1}$ via its identification with $S O(1, n-1)$.

\section{Algebraic structure of groups of type $P_{2}$}

Consider on $\mathbf{R}^{n+2}$ the quadratic form of index 2: $q=x_{0} x_{n}+x_{1} x_{n+1}+\ldots+x_{2}^{2}+x_{n-1}^{2}$. Let $P$ be the vectorial 2-plane generated by the elements of the canonical basis $e_{0}$ and $e_{1}$, and $P_{2}$ its isotropy group in the orthogonal group of $q$. To understand $P_{2}$, we shall first observe that it contains the following four subgroups, and then, $P_{2}$ is a semi-direct product of them:

1) A semi-simple of non compact type subgroup: $S \sim S L(2, \mathbf{R})$. We have the following representation of $S L(2, \mathbf{R})$ in $P_{2}$ :

$$
A=\left(\begin{array}{ll}
a & b \\
c & d
\end{array}\right) \in S L(2, \mathbf{R}) \rightarrow R_{A}=\left(\begin{array}{ccccc}
a & b & & & \\
c & d & & & \\
& & I & & \\
& & & d & -c \\
& & & -b & a
\end{array}\right) \in S \subset P_{2} .
$$

2) A semi-simple compact subgroup $R$ isomorphic to $S O(n-2)$. We have the following representation of $S O(n-2)$ in $P_{2}$ :

$$
A \in S O(n-2) \rightarrow R_{A}=\left(\begin{array}{ccccc}
1 & 0 & & & \\
0 & 1 & & & \\
& & A & & \\
& & & 1 & 0 \\
& & & 0 & 1
\end{array}\right) \in R \subset P_{2} .
$$

3) A diagonal subgroup $D$ isomorphic to $\mathrm{R}^{*}$ : 


$$
\lambda \in \mathbf{R}^{*} \rightarrow R_{\lambda}=\left(\begin{array}{ccccc}
\lambda & 0 & & & \\
0 & \lambda & & & \\
& & I & & \\
& & \lambda^{-1} & 0 \\
& & & 0 & \lambda^{-1}
\end{array}\right) \in D \subset P_{2} .
$$

4) We finally have a unipotent subgroup $N$ isomorphic to the Heisenberg group of dimension $2(n-2)+1$.

$$
\begin{gathered}
\left(a_{2}, \ldots, a_{n-1} ; b_{2}, \ldots, b_{n-1}, c\right) \rightarrow R_{\left(a_{2}, \ldots, a_{n-1} ; b_{2}, \ldots, b_{n-1}, c\right)} \\
=\left(\begin{array}{ccccc}
1 & 0 & 2 a_{2} \cdots 2 a_{n-1} & -\Sigma a_{i}^{2} & -c-2 \sum a_{i} b_{i} \\
0 & 1 & 2 b_{2} \cdots 2 b_{n-1} & c & -\Sigma b_{i}^{2} \\
0 & 0 & 1 \cdots & -a_{2} & -b_{2} \\
& & \cdots & \cdot & \cdot \\
& \cdots & \cdot & . \\
& \cdots & . & . \\
& \cdots 1 & -a_{n-1} & -b_{n-1} \\
& \cdots & 1 & 0 \\
& \cdots & 0 & 1
\end{array}\right)
\end{gathered}
$$

The adjoint action on $N$. One verifies that $N$ is a normal subgroup, and that the action by conjugacy on it is given as follows:

1. The action of $S$ : represent an element $R_{\left(a_{2}, \ldots, a_{n-1} ; b_{2}, \ldots, b_{n-1}, c\right)} \in N$ by $R_{\left(\left(a_{2}, b_{2}\right), \ldots,\left(a_{n-1}, b_{n-1}\right) ; c\right)}$. Then, for $A \in S L(2, \mathbf{R})$ :

$$
R_{A} R_{\left(\left(a_{2}, b_{2}\right), \ldots,\left(a_{n-1}, b_{n-1}\right) ; c\right)} R_{A}^{-1}=R_{\left(A\left(a_{2}, b_{2}\right), \ldots, A\left(a_{n-1}, b_{n-1}\right) ; c\right)}
$$

2. The action of $R$ : if $A \in S O(n-2)$, then

$$
R_{A} R_{\left(a_{2}, \ldots, a_{n-1} ; b_{2}, \ldots, b_{n-1}, c\right)} R_{A}^{-1}=R_{\left(A\left(a_{2}, \ldots, a_{n-1}\right) ; A\left(b_{2}, \ldots, b_{n-1}\right), c\right)}
$$

3. The action of $D$ :

$$
R_{\lambda} R_{\left(a_{2}, \ldots, a_{n-1} ; b_{2}, \ldots, b_{n-1}, c\right)} R_{\lambda}^{-1}=R_{\left(\lambda a_{2}, \ldots, \lambda a_{n-1} ; \lambda b_{2}, \ldots, \lambda b_{n-1}, \lambda^{2} c\right)}
$$

Fact 5.1 $P_{2}$ is a semi-direct product: $P_{2}=N \rtimes(S . D . R)$, where the factors $S, D$ and $R$ commute and act on $N$ as above. Furthermore D.N (resp. $N$ ) is the radical (resp. nilpotent radical) of $P_{2}$.

Action on $\mathbf{H}^{1, n}$ : $P_{2}$ acts transitively on $\mathbf{H}^{1, n}$, which therefore may be seen as a homogeneous space $P_{2} / I_{e}$, where we choose the base point $e \in \mathbf{H}^{1, n}$ to be $e=(0,1,0, \ldots, 0,-1)$. To compute the isotropy group $I_{e}$, let us introduce the following notations:

i) $N_{0}$, the center of $N: N_{0}=\left\{R_{(0, \ldots, 0 ; 0, \ldots, 0 ; c)}, c \in \mathbf{R}\right\}$.

ii) $N_{1}$ (resp. $N_{2}$ ) the "upper" (resp. "lower") "half" of $N$, isomorphic to the abelian group $\mathbf{R}^{n-2}: N_{1}=\left\{R_{\left(a_{2}, \ldots, a_{n-1} ; 0, \ldots, 0 ; 0\right)}, a_{i} \in \mathbf{R}\right\}$, and $N_{2}=$ $\left\{R_{\left(0, \ldots, 0 ; b_{2}, \ldots, b_{n-1}, 0\right)}, b_{i} \in \mathbf{R}\right\}$ 
iii) Observe that $N_{0} \rtimes(S . D)$ is a subgroup of $P_{2}$. It is in fact just the stabilizer in $P_{2}$ of the 4-space generated by $e_{0}, e_{1}, e_{n}$ and $e_{n+1}$. Notice also that $N_{0} \rtimes(S . D)$ is a direct product $S \times\left(D . N_{0}\right)$.

Let $U^{+} \subset S L(2, \mathbf{R})$ be the subgroup of upper triangular matrices. Both $U^{+}$ and $D . N_{0}$ are canonically isomorphic to $A G$, the group of affine transformations of the line. Let Diag be the diagonal of $U^{+} \times\left(N_{0} \rtimes D\right)$ :

$$
\operatorname{Diag}=\left\{R_{A} R_{\lambda} R_{(0, \ldots, 0 ; 0, \ldots, 0 ; c)} / A=\left(\begin{array}{cc}
\lambda & -c \lambda \\
0 & \lambda^{-1}
\end{array}\right), c \in \mathbf{R}, \lambda \in \mathbf{R}^{+}\right\}
$$

Fact $5.2 I_{e}=N_{1} \rtimes(\operatorname{Diag} \cdot R)$

\section{Main Proposition}

Main Proposition 6.1 The homogeneous space $\left(P_{2}, \widetilde{\mathbf{H}^{1, n}}\right)$ has no compact quotient (that is there is no compact quotient $\tilde{\Gamma} \backslash \widetilde{\mathbf{H}^{1, n}}$, with $\tilde{\Gamma} \subset \tilde{P}_{2}$ ).

Remark 6.2 In geometric terms, the Main Proposition is equivalent to Theorem $\mathbf{C}$, that is a compact anti de Sitter spacetime has no codimension 1 lightlike geodesic foliation. In algebraic terms, it means that the homogeneous space $\tilde{P}_{2} / \tilde{I}_{e}$ has no compact quotient (notation of Sect. 5 ).

\subsection{Preliminaries}

Notations. $\tilde{P}_{2}, \tilde{S}, \tilde{R}, \tilde{D}$ and $\tilde{N}$ are respectively, the identity components of the inverse images in $\operatorname{Isom}\left(\widetilde{\mathbf{H}^{1, n}}\right)$, of the groups $P_{2}, S, R, D$ and $N$. However, we omit the tilde over $D$ and $N$, because these later groups are simply connected. For the same reason we denote Diag, simply by Diag. As for $P_{2}$, we have a semi direct product $\tilde{P}_{2}=N \rtimes(\tilde{S} . \tilde{R} . D)$.

If $A$ is a group which is a factor in the semi-direct product structure of $P_{2}$ or $\tilde{P}_{2}$, then the projection onto $A$, will be denoted by $\operatorname{Proj}_{A}$.

Application of the Lemma 3.4. The adjoint action of $D$ on $N$ yields non trivial homotheties commuting with the action of $A=\tilde{S} . \tilde{R} . D$. Thus the lemma 3.4 is applicable to $\tilde{P}_{2}$.

Lie subgroups of $N$. Let $I=\left(i_{2}, \ldots, i_{d}\right), i_{j} \in\{2, \ldots, n-1\}$ be an ordered multi-index, and $\alpha_{I}=\left(\alpha_{i_{2}}, \ldots, \alpha_{i_{d}}\right), \alpha_{i_{j}}$ real, $\neq 0$. Consider $\Delta_{\alpha_{I}}$ the 3 - dimensional Heisenberg subgroup of $N$ generated by the center $N_{0}$ together with the "diagonal" elements of the form $\left(0, \ldots, \alpha_{i_{2}} x, \ldots, \alpha_{i_{d}} x, \ldots, 0\right) \in N$, for $x \in \mathbf{R}^{2}$.

Consider also the $2(d-1)+1$ Heisenberg subgroup Heis (which does not depend on $\alpha_{I}$ but only on $\left.I\right)$, generated by the $\mathbf{R}^{2}$ factors corresponding to the coordinates $x_{i_{2}}, \ldots, x_{i_{d}}$. 
One proves the following lemma in a standard way by considering irreducible invariant subspaces of the diagonal representation of $\operatorname{SL}(2, \mathbf{R})$ in $\left(\mathbf{R}^{2}\right)^{n-2}$.

Lemma 6.3 A connected subgroup of $N$, invariant by the $S L(2, \mathbf{R})$-adjoint action, is a (direct) product of some subgroups of type $\Delta_{I}$ or Heis I $_{I}$ associated to disjoint multi-indices. In particular such a subgroup is normal in $N$, contains $N_{0}$, and is non abelian unless it equals $N_{0}$.

\subsection{Steps}

Suppose by contradiction that such a quotient $M=\tilde{\Gamma} \backslash \widetilde{\mathbf{H}^{1, n}}$ exists. Thus the projection $\Gamma$ of $\tilde{\Gamma}$ is contained in $P_{2}$.

Step $1 L=\operatorname{proj}_{S}(\Gamma) \subset S$ is not solvable.

Proof. Assume the contrary. Suppose firstly that $L$ is not precompact. Every non precompact solvable subgroup of $S L(2, \mathbf{R})$ is conjugate to a subgroup of $U^{+}$, the group of upper triangular matrices (notation of the previous section). It then follows that, after conjugacy: $\Gamma \subset N \rtimes\left(U^{+} . D . R\right)$. But this last group preserves the line $\mathbf{R} e_{0}$, which is impossible by Sect. 4 .

Suppose now that $L$ is precompact. Then after conjugacy, $\Gamma$ is contained in $G^{\prime}=N \rtimes(K . D . R)$, where $K$ is the rotation group $\left\{\left(\begin{array}{cc}\cos (\theta) & -\sin (\theta) \\ \sin (\theta) & \cos (\theta)\end{array}\right)\right\}$. One verifies that $G^{\prime}$ acts transitively on $\mathbf{H}^{1, n}$, and that the isotropy group of the base point $e=(0,1,0, \ldots, 0,1)$ is now $N_{1} \rtimes R$. In particular $D$ normalizes this last isotropy group, and hence acts naturally by the right on $\mathbf{H}^{1, n}=N \rtimes(K . D . R) / N_{1} \rtimes$ $\tilde{R}$. We now conclude with the help of Proposition 3.1, by observing that $D$ has not the same modular distortion along $N \rtimes(K . D . \tilde{R})$ and along $N_{1} \rtimes \tilde{R}$.

Step $2 \tilde{L}=\operatorname{proj}_{\tilde{S}}(\tilde{\Gamma}) \subset \tilde{S}$ is discrete.

Proof. Let $\tilde{L}^{\prime}=\operatorname{proj}_{\tilde{S} . D . \tilde{R}}(\tilde{\Gamma})$, so $\tilde{L}=\operatorname{proj}_{\tilde{S} . D . \tilde{R}}\left(\tilde{L^{\prime}}\right)$. We argue by contradiction and consider many cases and subcases:

Case 1: $\tilde{L}^{\prime}$ is discrete.

Subcase 1: $\tilde{R}$ is compact, i.e. $n-2 \neq 2$ : Since $\tilde{R}$ is compact, $\operatorname{proj}_{\tilde{S} . D}\left(\tilde{L^{\prime}}\right)$ is also discrete. We apply Lemma 3.4, to this last projection, for $A=\tilde{S}$ and $N=D$. The projection onto $\tilde{S}$ is exactly $\tilde{L}$. So, if non discrete, $\tilde{L}$ would have a non trivial normal nilpotent subgroup. However, a subgroup of $\widetilde{S L(2, \mathbf{R})}$ (or $S L(2, \mathbf{R}))$ which has a normal non central nilpotent subgroup, is solvable. This contradicts the previous step.

Subcase 2: $n-2=2$, i.e. $\tilde{R}=\mathbf{R}$. We apply the same previous argument to $\tilde{S} . D . \tilde{R}$, which is now isomorphic to $\tilde{S} \times \mathbf{R}^{2}$.

Case 2: $\tilde{L}^{\prime}$ is not discrete.

Subcase 1: $\tilde{R}$ is compact. If the non-discrete part $\tilde{L}_{n d}^{\prime}$ which is nilpotent by 3.4 , projects non trivially on $\tilde{S}$, then it is as in the case 1 , solvable. 
If in contrary $\tilde{L}_{n d}^{\prime}$ is contained in $\tilde{R}$, then by compactness of $\tilde{R}$ and $3.3, \tilde{L}^{\prime}$ projects discretely on $\tilde{S} . D$. So, we are able to use 3.4 , with $A=\tilde{S} ; N=D$, and conclude that $\tilde{L}$ is discrete, since it is not solvable.

If now $\tilde{L}_{n d}^{\prime}$ is contained in $D . \tilde{R}$ but not in $\tilde{R}$, then it is co-compact in the "cylinder" $D . \tilde{R}$. From 3.3, we get that the projection of $\tilde{L}^{\prime}$ on $\tilde{S}$ is discrete.

Subcase 2: $n-2=2$, i.e, $\tilde{R}=\mathbf{R}$. Thus $\tilde{S} . D . \tilde{R} \sim \tilde{S} \times \mathbf{R}^{2}$. The same argument as above works if $\tilde{L}_{n d}^{\prime}$ is not contained in $\mathbf{R}^{2}$, or also, in contrary if it is cocompact in $\mathbf{R}^{2}$. The remaining case is when $\tilde{L}_{n d}^{\prime} \subset \mathbf{R}^{2}$ is isomorphic to $\mathbf{R}$. In that case, we divide by it (since it is normal) and get a discrete projection onto $\tilde{S} \times \mathbf{R}$. Again as above, we apply 3.4 to this last discrete group.

Step 3 Up to a change of $\tilde{\Gamma}$ by a subgroup of finite index, the projection $\tilde{L}^{\prime \prime}=$ $\operatorname{proj}_{D . \tilde{R}}(\tilde{\Gamma})$ is abelian.

Proof. In the case $n-2=2$, i.e. $\tilde{R}=\mathbf{R}$, there is nothing to prove, because $D . \tilde{R}$ itself is abelian. Now, let $n-2 \neq 2$, that is $\tilde{R}=R$ is compact. By the previous step, the non discrete part $\tilde{L}_{n d}^{\prime \prime}$ is nilpotent, because it coincides with the non discrete part of $\tilde{L}^{\prime}=\operatorname{proj}_{\tilde{S} . D .}\left(\tilde{R}(\tilde{\Gamma})\right.$ itself (since $\tilde{L}=\operatorname{proj}_{\tilde{S}}(\tilde{\Gamma})$ is discrete). Therefore $\tilde{L}_{n d}^{\prime \prime}$ is abelian, because any nilpotent, or even solvable subgroup of $D . \tilde{R}$ (which is isomorphic to $\mathbf{R} \times S O(n-2)$ ) is abelian.

The intersection $\tilde{L}^{\prime \prime} \cap \tilde{R}$ is of finite index in $\tilde{L}_{n d}^{\prime \prime} \cap \tilde{R}$ (because $\tilde{R}$ is compact). In order to have equality between these last subgroups, and in particular, to ensure that $\tilde{L}^{\prime \prime} \cap \tilde{R}$ is abelian, we just replace $\tilde{\Gamma}$ by its finite index subgroup $\operatorname{proj}_{\tilde{R}}^{-1}\left(\tilde{L}_{n d}^{\prime \prime} \cap \tilde{R}\right)$.

Observe that the derivative group $\left[\tilde{L}^{\prime \prime}, \tilde{L}^{\prime \prime}\right]$ is contained in $\tilde{L}^{\prime \prime} \cap \tilde{R}$ and is hence abelian. Therefore $\tilde{L}^{\prime \prime}$ is solvable and hence abelian.

Step 4 i) $L$ is Zariski dense in $S L(2, \mathbf{R})$ and after conjugacy, the Zariski closure $G$ of $\Gamma$ contains $S L(2, \mathbf{R})$ (we shall suppose that it is the case in what follows).

ii) $\tilde{\Gamma} \cap(N \rtimes(D . \tilde{R}))$ is infinite.

Proof. For the first point, observe that a non-solvable subgroup of $S L(2, \mathbf{R})$ is Zariski dense. Therefore, the algebraic closure of $G$ projects surjectively on $S L(2, \mathbf{R})$. Thus it contains a semi-simple subgroup isomorphic to $S L(2, \mathbf{R})$. But all such subgroups are conjugate in $P_{2}=N \rtimes(S . D . R)$, as maximal semi-simple subgroups of non-compact type [1].

The last point follows from a standard cohomological dimension argument. Assume that $\tilde{\Gamma} \cap(N \rtimes D . \tilde{R})$ is finite. After passing to a finite index subgroup we may suppose that $\tilde{\Gamma} \cap(N \rtimes D . \tilde{R})$ is trivial, that is $\tilde{\Gamma}$ injects into $\tilde{S}$ (as a discrete group by the previous steps). Since $\tilde{S}$ is homeomorphic to $\mathbf{R}^{3}$, this implies that the cohomological dimension of $\tilde{\Gamma}$ is $\leq 3$. Hence $\operatorname{dim} M \leq 3$ (because $\tilde{M}$ is contractible). But the present article deals only with higher dimensions!

Step 5 Let $G\left(\right.$ resp. $\left.G_{N}\right)$ be the algebraic closure of $\Gamma$ (resp. $\left.\Gamma \cap N\right)$. Then

(i) $\Gamma \cap N$ is a lattice in $G_{N}$, and $G_{N}$ is normalized by $\operatorname{SL}(2, \mathbf{R})$. In particular $G_{N}$ has the form described in Lemma 6.3. 
(ii) G contains $N$.

Proof. It is a general fact that a discrete subgroup of an unipotent group is a lattice in its algebraic closure. Thus $\Gamma \cap N$ is a lattice in $G_{N}$. Since $\Gamma$ normalizes the algebraic group $G_{N}$, then also the algebraic closure $G$ normalizes $G_{N}$ and hence also does so $S L(2, \mathbf{R})$ because it is contained in $G$.

The point (ii), that is $G$ contains $N$, follows from the co-compactness of the action of $G$ on $\mathbf{H}^{1, n}$. The unipotent radical of $G$ is a non trivial subgroup $N^{\prime} \subset N$; as described in Lemma 6.3. So, at "most", after conjugacy, $G$ equals $N^{\prime} \rtimes(S . D . R)$. It acts co-compactly on $\mathbf{H}^{1, n}=N \rtimes(S . D . R) / N_{1} \rtimes($ Diag.R $)$, if and only if $N_{1} \rtimes(\operatorname{Diag} . R)$ acts co-compactly on $N^{\prime} \rtimes(S . D . R) \backslash N \rtimes(S . D . R)$. This is easily seen to imply that $N_{1}$ acts co-compactly on $N / N^{\prime}$. But from the "symmetric" form of $N^{\prime}$ (Lemma 6.3), this happens only if $N^{\prime}=N$.

Step $6 \tilde{\Gamma} \cap N$ is trivial.

Proof. By contradiction, suppose that $\tilde{\Gamma} \cap N$ is not trivial. Notice thus that $\Gamma \cap N$ intersects non trivially $N_{0}$. Indeed if not, it projects injectively in $N / N_{0}=$ $\left(\mathbf{R}^{2}\right)^{n-2}$, and is in particular abelian. But this implies that also $G_{N}$ is abelian, which contradicts Lemma 6.3.

Now we check that $\tilde{\Gamma}$ is contained in $N \rtimes \tilde{S} . \tilde{R}$ (without $D$ ). Suppose that an element $\gamma=s d r n \in \tilde{\Gamma}$. Then the action of $\operatorname{Ad}(\gamma)$ on $N_{0}$, is reduced to that of $\operatorname{Ad}(d)$. Hence $\tilde{\Gamma} \cap N_{0}$, which is non trivial, is invariant by $\operatorname{Ad}(d)$. By discreteness, this implies $d$ is trivial.

Therefore we can restrict our group to $N \rtimes(\tilde{S} . \tilde{R})$, and since $\tilde{\Gamma} \cap N_{0}$ is cocompact in $N_{0}$, we can furthermore divide by $N_{0}$, to get a lower dimensional manifold. So the Heisenberg group $N$ is now replaced by $N^{\prime}=\left(\mathbf{R}^{2}\right)^{n-2}$. Our homogeneous space becomes: $N^{\prime} \rtimes(\tilde{S} . \tilde{R}) / N_{1} \rtimes(U . \tilde{R})$, where $U$ is the one parameter group of unipotent upper triangular matrices in $S L(2, \mathbf{R})$.

Observe that the one parameter group of diagonal matrices $\left(\begin{array}{cc}e^{t} & 0 \\ 0 & e^{-t}\end{array}\right)$ normalizes $N_{1} \rtimes(U . \tilde{R})$. Nevertheless, this one parameter group acts unimodularly on $N^{\prime} \rtimes(\tilde{S} . \tilde{R})$, but obviously non-unimodularly on $N_{1} \rtimes U . \tilde{R}$. This leads to a contradiction with Proposition 3.1, since our homogeneous space is supposed to have a (left) compact quotient.

End. It follows from the previous steps that $\operatorname{proj}_{D . \tilde{R}}$ maps injectively the infinite group $\tilde{\Gamma} \cap(N \rtimes D . \tilde{R})$ into an abelian subgroup of $D . \tilde{R}$. In particular $\Gamma \cap(N \rtimes D . R)$, and hence also its algebraic closure $H$, are abelian. But $H$ is normalized by $\Gamma$ and hence also by its algebraic closure $G$, that is $H$ is a normal subgroup of $G$. The abelian algebraic group $H$ has a Jordan decomposition $H=T . U$, where $T$ is diagonal, and $U$ is unipotent. In fact $T$ is contained in some conjugate of $D . R$ (in $N \rtimes D . R$ ) and $U$ is contained in $N$. Of course $T$ is not trivial, since otherwise $\tilde{\Gamma} \cap(N \rtimes D . \tilde{R})$ should be contained in $N$. 
The Zariski closure $\mathrm{G}$ normalizes each of the factors $T$ and $U$. Since it is contained in $G, N$ also normalizes $T$ and $U$. In fact $N$ centralizes $T$ because $N$ is normal in $N \rtimes D . R$. However, it follows from Sect. 5, that the adjoint action of $D . R$ on $N$. is faithful. This finishes the proof of the Main Proposition.

\section{Semi-simple groups acting co-compactly on $\mathbf{H}^{1, n}$}

The aim of this section is to prove the following proposition. The proof might be viewed so long by a specialist of Lie group theory. But, we believe that "geometrical" proofs are always interesting.

Proposition 7.1 Let $G$ be a proper semi-simple subgroup of $S O(2, n)$ acting cocompactly on $\mathbf{H}^{1, n}$. Then $G$ is conjugate to $S U(1, d)$, for $n=2 d$.

\subsection{Subgroups of $S O(1, n)$}

We prove the following fact in a standard way:

Fact 7.2 Let $H$ be a subgroup of $S O(1, n)$.

i) Assume that $H$ acts co-compactly on the hyperbolic space $\mathbf{H}^{n}$. Then $H$ is $S O(1, n)$ itself or is, up to a compact subgroup, a parabolic subgroup, fixing a point at infinity of $\mathbf{H}^{n}$, acting in fact transitively on $\mathbf{H}^{n}$.

ii) Assume that $H$ is self-adjoint, i.e. it is invariant under the transposition: $A \rightarrow A^{*}$. Then it is reductive (this is a general fact, valid for self-adjoint subgroups of $S L(N, \mathbf{R})$ ). Up to switching of coordinates (in $\left.(0) \times \mathbf{R}^{n+1}\right)$, the non compact part of $H$ is $S O(1, p)$ for some $p \leq n$.

Sketch of proof. It is a general fact, that if a reductive subgroup $H$ of a semisimple group $I$ of non compact type, acts co-compactly on the (Riemannian) symmetric space associated to $I$, then $H=I$.

(i) It suffices to consider the case where $H$ has a non compact radical, which therefore has exactly one or two fixed point at infinity, because it contains at least a parabolic or a hyperbolic element. Thus $H$ fixes a point at infinity, and hence it is contained in a parabolic subgroup. In fact $H$, up to a compact factor, equals this parabolic group.

(ii) In the case where $H$ is self-adjoint, it has one orbit (in $\mathbf{H}^{n}$ ) which is totally geodesic and may be thus identified to a subspace $\mathbf{H}^{p}$. We then apply the point (i).

We can assume that $G$ has no compact factor, since $G$ will still act cocompactly without these factors. In fact, any parabolic subgroup of $G$ acts cocompactly on $\mathbf{H}^{1, n}$, because it is co-compact in $G$. Observe finally that the problem is invariant by conjugacy.

Step 7 The action of $G$ on $\mathbf{H}^{1, n}$ is transitive. 
Proof. Let $P$ be a parabolic subgroup of $G$, it then acts co-compactly on $\mathbf{H}^{1, n}$. Let's show that there is a subgroup $P^{\prime}$ of $P$, acting co-compactly on $\mathbf{H}^{1, n}$ and preserving a lightlike geodesic hypersurface $H$. Indeed $P$ is contained in a maximal parabolic subgroup of $S O(2, n)$, i.e. a subgroup of type $\mathbf{P}_{1}$ or type $\mathbf{P}_{2}$. In the first case, we take $P^{\prime}=P$. In the other case, $P$ preserves a codimension 1 lightlike geodesic foliation, and hence it acts on its leaf space $S^{1}$, via a homomorphism $\alpha: P \rightarrow S L(2, \mathbf{R})$. If this action has no fixed point, then it factors through a rotation. We then take $P^{\prime}$ to be the Kernel of $\alpha$ which is co-compact in $P$, and therefore acts co-compactly on $\mathbf{H}^{1, n}$.

Let's show that $P^{\prime}$ acts transitively on $H$. As described in Sect. 4, $H$ has a natural 1-dimensional Riemannian foliation $\mathscr{D}$, with quotient space the hyperbolic space $\mathbf{H}^{n-1}$, and the action of $P_{1}$ on $\mathbf{H}^{n-1}$ factors through the usual action of its semi-simple factor $S_{1}$, which is isomorphic to $S O(1, n-1)$. Therefore the analogous action of $P^{\prime}$ factors via the action of $P^{\prime} \cap S_{1}$. Since $P^{\prime}$ acts co-compactly on $H, P^{\prime} \cap S_{1}$ acts co-compactly on $\mathbf{H}^{n-1}$, and hence transitively, by Fact 7.2. One easily sees this implies that $P^{\prime}$ itself acts transitively on $H$.

Let $x_{0} \in H$. Notice that the orbit $G x_{0}$ is not contained in $H$ since otherwise $G \subset P_{1}$, but any semi-simple subgroup of $P_{1}$ is conjugate to a subgroup of $S O(1, n-1)$, which does not act co-compactly on $\mathbf{H}^{1, n}$. Therefore $G x_{0}$ is open, since it is a connected submanifold containing strictly a closed codimension 1 submanifold $H$. Through each point of $G x_{0}$, passes (at least) a complete geodesic hypersurface, image of $H$. These images can not foliate, since otherwise $G$ will be contained in a parabolic group of type $P_{2}$, which is impossible for the same reasons as above. This fact may be used in a standard way to deduce that $G x_{0}$ can not have boundary points. That is $G$ acts transitively on $\mathbf{H}^{1, n}$.

Step 8 G has rank 1.

Proof. Recall that a higher rank semi-simple group has some parabolic subgroup $P$ containing a non compact semi-simple group. This $P$ is contained in a parabolic subgroup of $S O(2, n)$ of type $\mathbf{P}_{1}$ or $\mathbf{P}_{2}$. The argument for the two possibilities is the same, and so we will just consider the case $P$ of type $\mathbf{P}_{1}$, more precisely: $P \subset P_{1}$ (notation of Sect. 4).

As in the argument of the previous step, $P$ projects onto a subgroup of $S_{1} \sim S O(1, n-1)$, which acts transitively on $\mathbf{H}^{n-1}$, and contains a non trivial semi-simple group. Therefore, from 7.2, $P$ projects surjectively onto $S_{1}$ and hence, up to a conjugacy, $P$ contains $S_{1}$. Let $U$ be the unipotent radical of $P$. If $U=\{1\}$, then up to a conjugacy $P \subset S_{1} . D$ (notation of Sect. 4). But this later group does not act co-compactly on $\mathbf{H}^{1, n}$. Therefore $U$ is a non trivial subgroup of $\mathbf{R}^{n}$, invariant by $S_{1}$. This implies $U=\mathbf{R}^{n}$, and so $P$ contains $L=\mathbf{R}^{n} \rtimes S_{1}$. Let $\mathscr{C}$ be the symmetric space determined by $S O(2, n)$. The orbits of $L$ have at most codimension 1 , since $P_{1}=L . D$ acts transitively on $\mathscr{M}$ (the orbits of $L$ are in fact horospheres) As in the transitivity argument above, the orbits of $G$ which contain that of $L$ are open, since otherwise $G$ coincides with $L$, but by semi-simplicity, this implies: $G=S_{1}$. Therefore $G$ acts transitively on $\mathscr{C}$, 
which contradicts our hypothesis that $G$ is a proper semi-simple subgroup of $S O(2, n)$.

Step 9 The action of $G$ on $\mathbf{H}^{1, n}$ is proper.

Proof. Since $G$ acts transitively, to prove that it acts properly, we have just to prove that its intersection with the isotropy group $S O(1, n)$ is compact.

It is known, since $G$ is semi-simple that it has a conjugate (in $S O(2, n)$ ) which is self-adjoint, i.e., invariant under the transposition map $A \in G L(n+2) \rightarrow A^{*} \in$ $G L(n+2)$. Assume by contradiction that $I_{G}=G \cap S O(1, n)$ is not compact. It follows from 7.2, that, up to switching of coordinates, $I_{G}$ contains $S O(1, p)$ for some $p$. It contains in particular some $\mathbf{R}$-semi-simple one parameter group. To such a one parameter is associated a parabolic group $P$ of $G$.

It is easy to see that any $\mathbf{R}$-semi-simple one parameter group of $S O(1, n)$ is conjugate to the diagonal group $D_{1}$ of the group $P_{1}$, and hence up to conjugacy, $P=G \cap P_{1}$. Let $K$ be the centralizer of $D_{1}$ in $G$. It equals $S_{1} \cap P$, and is compact since $G$ has rank 1 . This contradicts the fact that $S_{1} \cap P$ must act co-compactly on the leaf space of the Riemannian foliation of the geodesic hypersurface $H$ associated to $P_{1}$.

End of the Proof. Since $G$ acts properly transitively on $\mathbf{H}^{1, n}$, a co-compact lattice in $G$ yields a compact quotient of $\mathbf{H}^{1, n}$. Hence, as mentioned in Sect. 1, $n=2 d$, that is $n$ is even. Let us first prove that $G$ is isomorphic to $S U(1, d)$.

Observe that $K=S O(2 d) \times S^{1}$ is a maximal compact subgroup of $S O(2, n)$, and denote by $\mathscr{K}$ and $\mathscr{T}$ their respective Lie algebras, and by $\mathscr{G}$ that of $G$. Hence $\mathscr{T}$ has a Cartan decomposition $\mathscr{T}=\mathscr{K}+\mathscr{P}$.

We assume as above that $G$ is self-adjoint, i.e. invariant under the transposition map $A \rightarrow A^{*}$. This implies in particular that $K_{G}=G \cap K$ is a maximal compact subgroup of $G$ and that the cartan decomposition above induces a similar one on $\mathscr{F}=\mathscr{G} \cap \mathscr{K}+\mathscr{G} \cap \mathscr{P}$. Recall that $\mathscr{P}$ and $\mathscr{P} \cap \mathscr{G}$ are identified with the tangent spaces of the corresponding symmetric spaces (at points with isotropy, respectively $K$ and $K_{G}$ ).

If $K$ projects trivially on the factor $S^{1}$ of $K$, then $K \subset S O(2 d) \subset S O(1,2 d)$. That is $K_{G}=I_{G}=G \cap S O(1, n)$. Hence $\mathbf{H}^{1, n}=G / K_{G}$ is canonically identified to the symmetric space associated to $G$. However the symmetric space determined by a simple Lie group, without compact factor, is simply connected [20], but this is not the case of $\mathbf{H}^{1, n}$.

Therefore, $K_{G}$ projects non trivially on $S^{1}$. Thus $K_{G}$ contains a factor isomorphic to $S^{1}$. It is known that this implies that $G$ determines a Kahlerian symmetric space [20]. This is precisely a complex hyperbolic space, since $G$ has rank one. This space has dimension $\operatorname{dim}\left(\mathbf{H}^{1,2 d}\right)-1$. Hence $G$ is isomorphic to $S U(1, d)$.

We have to prove now that $G$ is in fact conjugate to $S U(1, d)$. The tangent space $\mathscr{P}$ of the symmetric space associated to $S O(2,2 d)$ ( at a base point with 
isotropy, exactly $\left.S O(2 d) \times S^{1}\right)$ is identified to $\mathbf{R}^{2 d} \oplus \mathbf{R}^{2 d}=\mathbf{R}^{2 d}+i \mathbf{R}^{2 d}=\mathbf{C}^{2 d}$, endowed with the complexified action of $S O(2 d)$ together with the action of $S^{1}$ by complex multiplication.

The symmetric space associated to $G$ is the geodesic subspace tangent to $\mathscr{P}^{\prime}=\mathscr{G} \cap \mathscr{P}$. Since it is isometric to the complex hyperbolic space of real dimension $2 d, \mathscr{P}^{\prime}$ is isomorphic to a $2 d$ subspace of $\mathscr{P}$. Moreover, the action of $K_{G}$ on $\mathscr{P}^{\prime}$ is conjugate to the usual action of $S U(d) \times S^{1}$ on $\mathbf{C}^{d}$.

Notice that for $G=S U(1, d)$, the corresponding $\mathscr{P}^{\prime}$ is: $\{x+i \sqrt{-1} x \quad x \in$ $\mathbf{R}^{2 d}$, where $\mathbf{R}^{2 d}$ is canonically identified with $\mathbf{C}^{d}$.

We shall prove that after conjugacy by an element of $K, \mathscr{P}^{\prime}=\{x+i \sigma(x) \in$ $\left.\mathbf{R}^{2 d}+i \mathbf{R}^{2 d}, \quad x \in \mathbf{R}^{2 d}\right\}$, for some $\sigma: \mathbf{R}^{2 d} \rightarrow \mathbf{R}^{2 d}$ conjugate to the multiplication by $\sqrt{-1}$. That conjugacy sends $\mathscr{P}^{\prime}$ to $\left\{x+i \sqrt{-1} x \quad x \in \mathbf{R}^{2 d}\right\}$. . This means in geometric words that the geodesic subspace determined by $G$ is congruent to that determined by $S U(1, d)$. Thus $G$ is conjugate to $S U(1, d)$.

Write $K_{G}=S \times R$, where $S$ is isomorphic to $S U(d) \subset S O(2 d)$ and $R$ projects one to one on $S^{1}$. Notice that $\mathscr{P}^{\prime}$ is not contained in a factor $\mathbf{R}^{2 d}$ or $i \mathbf{R}^{2 d}$, since $K_{G}$ contains elements outside $S O(2 d)$. Therefore the projection of $\mathscr{P}^{\prime}$ onto $\mathbf{R}^{2 d}$ is not trivial, and in fact conjugate (bijectively) the action of $S$ to an action of a subgroup $S^{\prime}$ of $S O(2 d)$ (this follows from the equality of dimensions and the fact that the action of $S$ is irreducible ). Therefore $\mathscr{P}^{\prime}=\left\{x+i \sigma(x) \in \mathbf{R}^{2 d}+i \mathbf{R}^{2 d}, x \in\right.$ $\mathbf{R}^{2 d}$, for some $\sigma: \mathbf{R}^{2 d} \rightarrow i \mathbf{R}^{2 d}$, commuting with the action of $S^{\prime}$.

Take an element of $R$ of the form $(f, i)$. Since it preserves $\mathscr{P}^{\prime}$, we get: $\sigma f \sigma=-f$. Both of $f$ and $\sigma$ centralize $S^{\prime}$, and so they commute, since the centralizer of $S^{\prime}$ is abelian (because $S^{\prime}$ is irreducible). Hence: $\sigma^{2}=-1$. That is $\sigma$ defines a complex structure, with isotropy group contained in $S O(2 d)$. Therefore $\sigma$ is conjugate in $S O(2 d)$ to the multiplication by $\sqrt{-1}$ on $\mathbf{R}^{2 d}$.

Therefore, with the notations of 4 , the projection of $P^{\prime}$ on $S O(1, n-1)$ acts co-compactly on $\mathbf{H}^{n-1}$. From 7.2, this projection acts in fact transitively on $\mathbf{H}^{n-1}$, the quotient space of $\mathscr{D}$. It is easy to show that the stabilizer in $P^{\prime}$ of a leaf of $\mathscr{D}$ must act transitively on that leaf. That is $P^{\prime}$ acts transitively on $H$.

This finishes the proof of Proposition 7.1.

\section{Standard spacetimes. Proofs of Theorems A and B.}

The following result gives equivalent characterizations of standard spacetimes.

Proposition 8.1 Let $M=\tilde{\Gamma} \backslash \widetilde{\mathbf{H}^{1, n}}$ be a compact anti de Sitter spacetime. Then the following conditions are equivalent:

i) $M$ has a non trivial timelike Killing field.

ii) $M$ has a non trivial Killing field, i.e. $\operatorname{Isom}^{0}(M)$ is not trivial.

iii) $\operatorname{Isom}^{0}(M)=S^{1}$.

iv) $\Gamma$ is conjugate to a lattice of $U(1, d)(n=2 d)$.

v) $M$ is standard.

Proof. From Fact 2.5 and the Main Proposition 6.1, if $M$ has a Killing field, i.e. $\Gamma$ centralizes some one parameter group, then it is conjugate to a subgroup of 
$U(1, d)$. In fact it is Zariski dense in $S U(1, d)$ or $U(1, d)$ and so its centralizer coincides with that of $U(1, d)$ itself, which is $S^{1}$. This shows the equivalence of the conditions i, ii and iii, whenever we observe that the Killing field determined by $S^{1}$ is timelike.

For a standard manifold, $\Gamma$ has a finite index normal subgroup $\Gamma^{\prime}$ conjugate in $U(1, d)$. Hence $\Gamma$ normalizes the algebraic closure of $\Gamma^{\prime}$, which is $U(1, d)$ or $S U(1, d)$. But the normalizer of these two groups is exactly $U(d, 1)$, and hence $\Gamma \in U(1, d)$. Therefore $\Gamma$ is centralized by $S^{1}$, and so $M$ possesses a Killing field. That is from above: $\operatorname{Isom}^{0}(M)=S^{1}$.

It remains now to prove the converse, that is, if $\Gamma$ is contained in $U(1, d)$, then $M$ is standard. It suffices to show that $\Gamma$ that $\Gamma$ is discrete. fact: if $\tilde{\Gamma} \subset$ $\widehat{U(1, d)}=S U(1, d) \times \mathbf{R}$ is a co-compact lattice, then its projection $\Gamma$ in $U(1, d)=$ $S U(1, d) \times S^{1}$ is discrete (and hence it is a lattice). It suffices for this to show that $\tilde{\Gamma}$ cuts non trivially the factor $\mathbf{R}$. To ensure this, we have just to check that the projection $\tilde{\Gamma}_{0}$ of $\tilde{\Gamma}$ on $S U(1, d)$ (as a factor of $\left.\widetilde{U(1, d)}\right)$ ) is discrete. But if this was not the case, then this projection would have a non trivial non discrete part, which is nilpotent by Lemma 3.4. Thus $\tilde{\Gamma}_{0}$ normalizes a non trivial nilpotent group, and is therefore contained in a parabolic group $P$. Hence $\tilde{\Gamma}$ is a co-compact lattice of $P \times \mathbf{R}$, but this is impossible, because the later group is not unimodular.

\subsection{Proofs of Theorems A and B}

Let $M=\tilde{\Gamma} \backslash \widetilde{\mathbf{H}^{1, n}}$ be a compact anti de Sitter spacetime. As explained in Sect. 3, $\operatorname{Isom}(M)$ is compact. Therefore, $\operatorname{Isom}(M)$ is finite, unless $M$ admits a non trivial Killing field, and thus $M$ is standard, by Proposition 8.1. By the same proposition, $\operatorname{Isom}(M)=S^{1}$, if $M$ is standard. This proves Theorem $\mathbf{B}$.

From 2.4, 6.1 and 7.1, the algebraic closure of $\Gamma$ is conjugate to $S O(2, n)$, $U(1, d)$ or $S U(1, d)$ (for $n=2 d$ ). From 8.1, $M$ is standard if $\Gamma$ is contained in $U(1, d)$. This proves the statement about the Zariski closure of $\Gamma$.

It remains to prove the finiteness of the level of $M$. Observe that this follows from the true definition when $M$ is standard. On the other hand, if $M$ is not standard, then $\operatorname{Isom}(M)$ is finite. The fundamental group of $\mathbf{H}^{1, n}$ is infinite cyclic, generated by a transformation $f \in \operatorname{Isom}\left(\widetilde{\mathbf{H}^{1, n}}\right)$, say. Because it is central, $f$ induces an isometry of $M$, which is of finite order since $\operatorname{Isom}(M)$ is finite. Therefore some power of $f$ belongs to $\tilde{\Gamma}$. This is easily seen to be equivalent to the finiteness of the level of $M$.

\section{References}

1. Borel, A.: Linear algebraic groups. Proc. Symp. Pure. Math. 9, Amer. Math. Soc, Providence, R.I, 3-19 (1966)

2. Calabi, E., Markus, L.: Relativistic space forms. Ann. Math. 75, 63-76 (1962) 
3. Carrière, Y.: Autour de la conjecture de L. Markus sur les variétés affines. Invent. Math. 95, 615-628 (1989)

4. Carrière, Y., D'Albo, F.: Généralisations du $1^{e r}$ Théorème de Bieberbach sur les groupes cristalographiques. Enseignement Math. 35, 245-262 (1989)

5. D'Ambra, G., Gromov, M.: Lectures on transformation groups : geometry and dynamics, Surveys in Differential Geometry (Supplement to the J. Differential Geom.) 1, 19-111 (1991)

6. Fried, D., Goldman, W.: Three dimensional affine criystallographic groups. Adv. Math. 47, 1-49 (1983)

7. Fried, D., Goldman, W., Hirsch, M.: Affine manifolds with nilpotent holonomy. Comment. Math. Helv. 56, 487-523 (1981)

8. Goldman, W., Kamishima, Y.: The fundamental group of a compact Lorentz space is virtually policyclic. J. Differential. Geom. 19, 233-240 (1984)

9. Grunewald, F., Margulis, G.: Transitive and quasi-transitive actions of affine groups preserving a generalized Lorentz structure. J. Geom. Phys. 5, 493-530 (1988)

10. Goldman, W.: Nonstandard Lorentz space forms. J. Differential. Geom. 21, 301-308 (1985)

11. Klingler, B: Complétude des variétés lorentziennes à courbure constante. Math. Ann. 306, 353370 (1996)

12. Kobayashi, T.: Proper action on a homogeneous space of reductive type. Math. Ann. 285, 249263 (1989)

13. Kulkarni, R., Raymond, F.: 3-dimensional Lorentz space- forms and Seifert fiber spaces. J. Diff. Geom. 21, 231-268 (1985)

14. Kulkarni, R.: Proper actions and pseudo-Riemannian space forms. Adv. Math. 40, 10-51 (1981)

15. Mess, G.: Lorentz spacetimes of constant curvature. Preprint, IHES

16. Millson, J.: A remark on Raghunathan's vanishing theorem. Topology 24, 495-498 (1985)

17. Raghunathan, M. S.: On the first cohomology of discrete subgroups of semi-simple Lie groups. Amer. J. Math. 87, 103-139 (1985)

18. Raghunathan, M. S.: Discrete subgroups of Lie groups. Berlin Heidelberg New York, SpringerVerlag, 1972

19. Salein, F.: Variétés anti-de Sitter de dimension 3 possédant un champ de Killing non trivial. C. R. Acad. Sci. Paris 324 Série I, 525-530 (1997)

20. Wolf, J.: Spaces of constant curvature. New York, McGraw-Hill, 1967

21. Zeghib, A.: Geodesic foliations in Lorentz 3-manifolds. Preprint, ENS-Lyon, 1994

22. Zeghib, A.: Sur le bord du groupe d'isométries d'une variété lorentzienne compacte. C. R. Acad. Sci. 322, Série I, 149-154 (1996) 